\title{
DETERMINANTES SOCIALES DE LA SALUD, PREDICTORES DE LA CONDICIÓN FÍSICA EN ADOLESCENTES ESCOLARES
}

Recibido: junio del 2018

Aceptado: marzo del 2019

Brayan Esneider Patiño Palma ${ }^{1}$, Miryam Tabares Gutiérrez², Consuelo Vélez Alvarez ${ }^{3}$, José Armando Vidarte Claros ${ }^{4}$

\section{Resumen}

Introducción. La actividad física $(\mathrm{AF})$ es un factor que condiciona la salud. En adultos, la falta de $\mathrm{AF}$ es un determinante de riesgo cardiovascular; en los niños, la evidencia sobre sus efectos es menor, sin embargo, existe relación con el desarrollo de obesidad y con factores de riesgo cardiovascular. Objetivo. Establecer los determinantes sociales de la salud como predictores de la condición física saludable de adolescentes escolares. Metodología. Estudio de tipo descriptivotransversal con una muestra de 339 estudiantes seleccionados aleatoriamente. Se aplicó la batería Alpha Fitness para determinar la condición física, además del cuestionario sobre determinantes. Resultados. El 84,4\% de los escolares presentaron condición física no saludable; los determinantes que mostraron asociación con la condición física fueron el nivel educativo del padre además del cigarrillo. Conclusión. Prevaleció la condición física no saludable en los escolares, donde el nivel educativo del padre y el consumo de cigarrillo fueron las variables predictoras.

Palabras clave: aptitud física, determinantes sociales de la salud (DeCS), ejercicio, estudiantes.

${ }^{1}$ Docente del programa de Fisioterapia de la Universidad de Boyacá. Miembro del grupo de investigación CORPS. Correo electrónico: bepatino@uniboyaca.edu.co

${ }^{2}$ Licenciada en educación física, magíster en Actividad Física y Deporte de la Universidad Autónoma de Manizales, miembro del grupo de investigación cuerpo y movimiento. Correo: mirtagu2@hotmail.com

2 Enfermera, doctora de la Universidad Andina Simón Bolívar. Docente del Departamento de Salud Pública de la Universidad Autónoma de Manizales. Miembro del grupo de investigación CuerpoMovimiento. Correo electrónico: cva@autonoma.edu.co

${ }^{2}$ Licenciado en educación física, doctor de la Universidad de la Coruña. Coordinador del grupo de investigación cuerpo y movimiento de la Universidad Autónoma de Manizales. Correo: cmovimiento@ manizales.autonoma.edu.co 


\section{DETERMINANTES SOCIALES DE LA SALUD, PREDICTORES DE LA CONDICIÓN FÍSICA EN ADOLESCENTES ESCOLARES}

Brayan Esneider Patiño Palma ${ }^{1}$, Miryam Tabares Gutiérrez², Consuelo Vélez Alvarez ${ }^{3}$, José Armando Vidarte Claros ${ }^{4}$

\section{Abstract}

Introduction: Physical activity (AF in Spanish) is a factor that subdues health. In adults, the lack of AF is a determinant in cardiovascular risk; in children, the evidence of its effects is less, although there is a relationship between the development of obesity with factors of cardiovascular risk. Objective: To establish social determinants of health as predictors of a physical condition in school teenagers. Method: Descriptive and cross-sectional study with a sample of 339 previously selected students. We applied the Alpha Fitness guide to determine physical condition and a questionnaire about determinants. Results: $84.4 \%$ of the school teenagers had an unhealthy physical condition. The determinants associated with the physical condition were the educational level of the father and smoking. Conclusions: An unhealthy physical condition was prevalent in the school teenagers. The educational level of the father and smoking were the predictive variables. 


\section{DETERMINANTES SOCIAIS DA SAUUDE, PREDITORES DA CONDIÇÃO FISICA EM ADOLESCENTES}

Brayan Esneider Patiño Palma' ${ }^{1}$ Miryam Tabares Gutiérrez², Consuelo Vélez Alvarez ${ }^{3}$, José Armando Vidarte Claros ${ }^{4}$

\section{Resumo}

Introdução: a atividade física (AF) é um fator que condiciona a saúde. Em adultos, a falta dela é um de determinante de risco cardiovascular; nas crianças, a evidência sobre seus efeitos é menor; contudo, existe relação com o desenvolvimento da obesidade e com fatores de risco cardiovascular. Objetivo: estabelecer os determinantes sociais da saúde como preditores da condição física saudável em adolescentes escolares. Metodologia: estudo de tipo descritivo-transversal com uma amostra de 339 estudantes selecionados aleatoriamente. Foi aplicada a bateria Alpha Fitness para determinar a condição física, além do questionário sobre determinantes. Resultados: 84,4 \% dos adolescentes apresentaram condição física não saudável; os determinantes que mostraram associação com a condição física foram o nível educativo do pai e o tabagismo. Conclusões: prevaleceu a condição física não saudável nos escolares, em que o nível educativo do pai e o consumo de cigarro foram as variáveis preditoras. 


\section{Introducción}

Uno de los grandes problemas de la salud pública en la actualidad es el aumento de la prevalencia de la obesidad $(1,2)$, especialmente en la región de las Américas, donde hoy en día alcanza en proporciones epidémicas y se resalta que más del $60 \%$ de los adultos tienen sobrepeso (3). Por su parte, la obesidad infantil está determinada no solo por factores ambientales, sino también hereditarios, sociales y familiares (4).

Se destaca especialmente el cambio en los estilos de vida que hoy mantienen los niños, pues estos no son los más activos, dado que se ha observado un aumento en las actividades sedentarias (como ver televisión), lo que aumenta los factores predisponentes al sobrepeso (5). Esto pone en evidencia la importancia y la necesidad de promover diferentes procesos en pro del desarrollo motriz, a partir del fomento de la actividad física y el desarrollo de la condición física saludable.

La actividad física es un factor de primera magnitud entre los que condicionan la salud humana (6). En los adultos, la falta de esta se convierte en un determinante del riesgo de mortalidad cardiovascular (7-9), sin embargo, es importante resaltar que el tiempo dedicado a las actividades sedentarias influye tanto en la salud como en el nivel de la actividad física, de hecho, se han observado asociaciones nocivas del tiempo dedicado a la televisión con los marcadores cardiometabólicos, incluso, en los individuos que cumplen lo establecido en las directrices de la OMS para la actividad física (10), por tanto, el abandono de la actividad física y el aumento de las actividades sedentarias durante la adolescencia hace más probable la obesidad en el adulto (11), sin embargo, hoy en día existe la tendencia de disminuir la actividad física de los adolescentes debido a múltiples factores como los currículos escolares, pues se evidencia un bajo cumplimiento de los estándares curriculares por parte de los profesores en la formación escolar (12), por las reglas de los padres relativas a la seguridad y las limitaciones del ambiente físico (13).

Ciertamente, la edad escolar y la adolescencia, son etapas cruciales para la configuración de hábitos alimenticios y otros estilos de vida, pues el desarrollo de estos durante la infancia perdurarán con más facilidad durante la edad adulta $(14,15)$. En la literatura existen numerosos estudios que señalan que la historia previa de ejercicio es un factor importante en el inicio o el mantenimiento de actividad física y deportiva, al observar cómo el alto nivel de actividad física de los sujetos en edades tempranas se ve reflejado en sus procesos motrices en edades adultas (16-19).

Lo anterior permite plantear cómo un buen desarrollo de la condición física se establece desde tempranas edades, al tener como principal herramienta la completa y correcta aplicación del currículo de enseñanza, al posibilitar así un ideal desempeño y desenvolvimiento psicológico, motor y social del niño $(12,20,21)$. Por esta razón, el análisis de las condiciones de salud y de vida, con el denominado enfoque de los determinantes sociales y económicos, es un tema central 
en la agenda de la OMS y de los ministerios de salud de varios países $(5,22)$.

En concordancia con lo anterior, el estudio de los determinantes sociales de la salud en las diferentes etapas de la vida, más que una dimensión es un enfoque útil para la investigación de las desigualdades sociales, ya que abarca los hechos sociales ajenos al individuo que pueden afectar su condición de salud y en este caso la condición física a lo largo de la vida (23); sin embargo, los estudios realizados hasta el momento (24-30) se han hecho con énfasis en variables nutricionales y su relación con las capacidades físicas, a pesar de ello, pocos evidencian propuestas desde los determinantes sociales y menos aún desde baterías motrices específicas para este grupo poblacional.

Igualmente, en el contexto regional y local aún no se realizan estudios que permitan establecer desde el análisis del comportamiento de los sujetos, asociaciones entre las variables sociales y la condición física saludable, lo que deja de lado procesos que ayuden a establecer predicciones de la condición física, es por ello que en la presente investigación se pretende establecer un modelo predictivo de la condición física saludable en escolares de 12 a 18 años, de la ciudad de Dosquebradas, a partir de la interrelación con los determinantes sociales de la salud.

\section{Materiales y métodos}

El presente estudio se consideró de tipo descriptivo-transversal con una fase comparativa, se seleccionó como pobla- ción al total de escolares registrados en la base de datos de la Secretaria Municipal de Educación para el 2016, escolares que debían estar entre 12 y 18 años, matriculados en colegios públicos y privados de la zona urbana de Dosquebradas, Risaralda.

Se realizó un muestreo de tipo probabilístico (muestreo aleatorio simple) donde se tuvo en cuenta para el tamaño de la muestra los estimadores reportados por Salleg y Petro31 de media y desviación estándar de las variables fuerza, resistencia abdominal, flexibilidad y $\mathrm{VO}^{2}$ máx.

La muestra seleccionada fue de 339 alumnos entre 12 y 18 años, incluyendo el ajuste de pérdida del $10 \%$, esta fue obtenida del promedio de los tres tamaños muéstrales realizado a partir de los estimadores anteriormente mencionados, los cuales fueron calculados con una confiabilidad del $95 \%$ y un margen de error de 0,6 cm para la variable de flexibilidad, una repetición para la resistencia abdominal y $1 \mathrm{ml} / \mathrm{kg} / \mathrm{min}$ para la variable $\mathrm{VO}^{2}$ máx.

La recolección de la información se realizó en instituciones tanto públicas como privadas, seleccionando a los escolares de manera proporcional en cuanto a la edad y el género. Los sujetos seleccionados para la investigación cumplieron con los siguientes criterios de inclusión y exclusión:

- Estudiantes con las edades establecidas en el estudio que se encuentren matriculados en las instituciones educativas. 
- Diligenciamiento del consentimiento informado por parte de los padres de familia o tutor legal y asentimiento por los escolares participantes del estudio.

- Al momento de la prueba física no estar apto o presentar alguna patología que impida su desarrollo.

- El colegio seleccionado para la evaluación de los estudiantes debe contar con 50 o más alumnos matriculados y registrados en la Secretaria de Educación Municipal.
Se emplearon preguntas estructuradas y semiestructuradas con las cuales se indagó sobre los determinantes individuales y sociales de la salud. Para la medición de la condición física relacionada con la salud, se siguió todo el protocolo establecido por la batería Alpha Fitness, versión extendida, determinándose de esta manera los cuatro principales componentes de la condición física saludable $(32,33)$ y se establecieron a partir de estos la condición física general en lo escolares evaluados (figura 1).

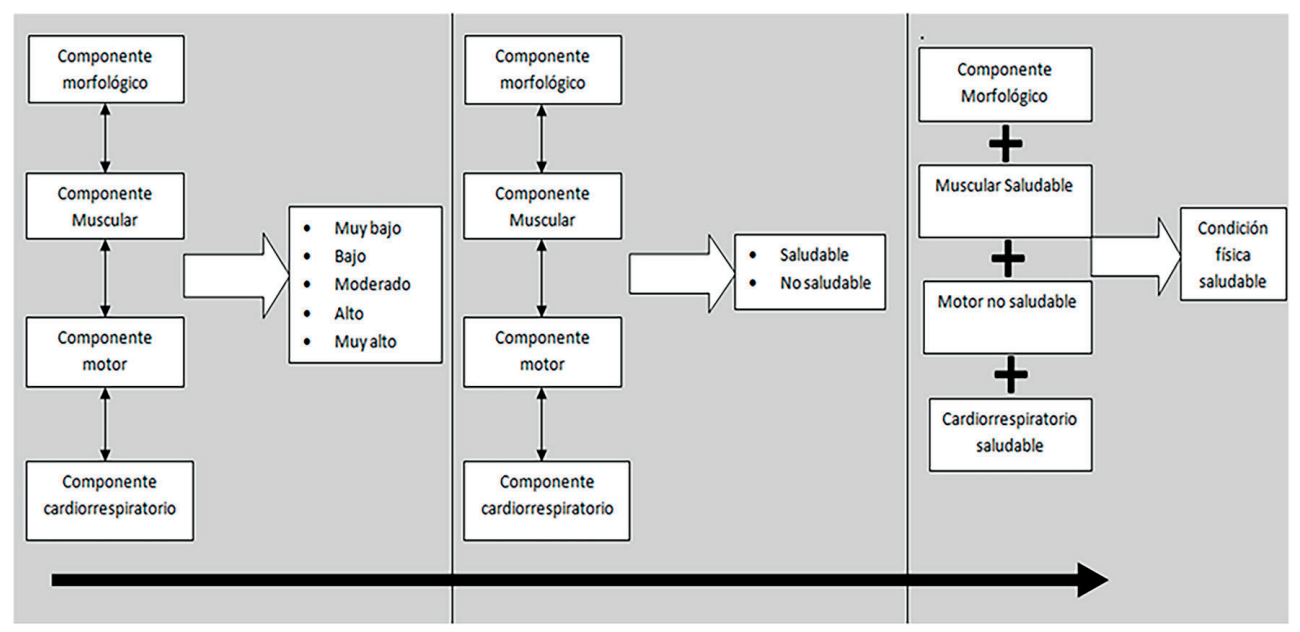

Figura 1. Proceso de recodificación de la condición física Fuente: elaboración propia.

El proceso consistió en recodificar en variables dummy (saludable y no saludable), el baremo establecido por el protocolo Alpha Fitness se consideró saludable en el nivel moderado en las tres pruebas que integran el componente morfológico; por su parte, para las pruebas que establecen el componente muscular y cardiorrespiratorio se consideró como saludable los niveles mo- derado, alto y muy alto; finalmente para el componente motor se determinaron como saludables los niveles moderado, bajo y muy bajo. Una vez recodificada la condición física por componentes se prosiguió a realizar una sumatoria de cada uno de estos, seguidamente, los autores establecen que un escolar al presentar uno o ningún componente no saludable, este debía ser catalogado con 
condición física saludable, pues Moro, et al. (34) menciona que la condición física es saludable siempre y cuando el sujeto evaluado presente buen desempeño en cuatro de los cinco componentes de la condición física relacionada con la salud.

Para la medición de las pruebas se utilizó un dinamómetro digital con agarre ajustable marca Camry, modelo EH101 con capacidad máxima hasta 90 kilogramos o 198 libras, el cual se calibró cada 100 evaluaciones, un decámetro $1 / 2$ $\times 50 \mathrm{~cm}$ de fibra de vidrio marca Stanley, una báscula electrónica marca Tanita Hd-314 calibrada cada 150 evaluaciones, un tallímetro portátil de pared marca Height de 0 a $200 \mathrm{~cm}$ de rango y precisión de $1 \mathrm{~mm}$ y un adipómetro marca Slim Guide calibrado cada 100 evaluaciones.

Para el plan de análisis se utilizó el software estadístico SPSS versión 24, licenciado por la Universidad Autónoma de Manizales, se realizaron pruebas de estadística descriptiva en las que se evaluó la frecuencia y la proporción de las variables cualitativas, así como las medidas de tendencia central y de variabilidad o dispersión para las variables cuantitativas. El análisis bivariado se desarrolló a partir de las relaciones encontradas entre la variable de estudio y la condición física saludable. Para determinar la significancia estadística de las relaciones resultantes, se aplicó la prueba estadística chi cuadrado, establecida a partir de las características propias de las variables categóricas. Para el análisis multivariado se realizó la construcción de un modelo de regre- sión binario no lineal tipo logit, el cual determinó a través de las diferentes variables del estudio, la relación entre estas y el hecho en cuestión, el cual fue determinado a partir de la significancia estadística de cada uno de los determinantes evaluados.

\section{Consideraciones éticas}

El presente estudio se consideró como “investigación con riesgo mínimo" de acuerdo al artículo 11 de la Resolución 008430 de 1993 del Ministerio de Salud colombiano (35), ya que se emplearon pruebas de evaluación no invasivas que no atentaron contra la integridad física y moral de los escolares participantes del estudio. Este fue aprobado según acta n. ${ }^{\circ} 62$ del 15 de febrero del 2017 del Comité de Bioética de la Universidad Autónoma de Manizales.

Adicionalmente, esta investigación cumplió con los principios enunciados en la Declaración de Helsinki de la Asociación Médica Mundial (36). Por otra parte, se respetaron los derechos de autor de los diferentes insumos teóricos y evaluaciones utilizadas al citar las respectivas referencias bibliográficas.

\section{Resultados}

De los escolares evaluados, el 50,4\% correspondieron al género femenino; de igual manera, más de la mitad de los escolares se encontraban en rango de edad entre 15 y 18 años y se destaca que la que el transporte activo (bicicleta 3,5\% y caminar 56,3\%) es el más común entre los estudiantes evaluados. 
Con relación a los hábitos saludables, más del $50 \%$ de los escolares evaluados mencionaron practicar ejercicio o actividad física, sin embargo, tan solo el $6 \%$ de estos estudiantes cumplen con la recomendación dada por la OMS de realizar actividad física mínimo 150 minutos por semana (37).

Se resalta que existe una baja prevalencia con relación al hábito el cigarrillo $(4,1 \%)$, sin embargo, en lo que corresponde al consumo de alcohol se observó presente en más de un tercio de los escolares.

Así mismo, sobresale que el estrato medio y el régimen de salud subsidiado fueron las variables de mayor proporción en los evaluados, de igual manera, se resalta que un tercio de los escolares mencionaron que sus padres se encuentran separados, destacando que el 86,7\% de los evaluados mencionaron vivir con la mamá, mostrando así que prevalecen las familias monoparenterales en la población estudiantil.

En la figura 2 se muestran los niveles de condición física, discriminados por cada uno de sus componentes, donde se destaca que más del $60 \%$ de los escolares evaluados presentaron condición física no saludable en tres de los cuatro componentes que evalúa la batería Alpha Fitness.

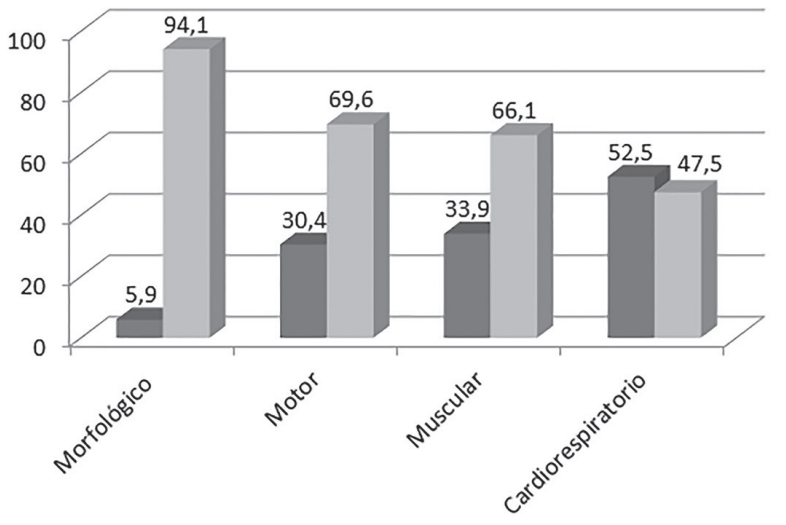

a Condición Fisica Saludable a Condición Fisica No Saludable

Figura 2. Condición física por componentes Fuente: elaboración propia.

Finalmente, y después de realizar la sumatoria de los componentes, se observa que la prevalencia observada de la con- dición física no saludable en los escolares entre 12 y 18 años de Dosquebradas, Risaralda, fue del $84,4 \%$. 
Tabla 1. Asociaciones entre determinantes de la salud y la condición física

\begin{tabular}{|c|c|c|c|c|c|}
\hline \multicolumn{3}{|c|}{ Determinantes sociales } & \multicolumn{3}{|c|}{ Determinantes individuales } \\
\hline Determinante & $x^{2}$ & P valor & Determinante & $X^{2}$ & P valor \\
\hline Tipo de colegio & 0,532 & 0,466 & Sexo & 0,006 & 0,937 \\
\hline Estrato & 2,302 & 0,316 & Edad & 0,126 & 0,723 \\
\hline $\begin{array}{l}\text { Afiliación al } \\
\text { sistema de salud }\end{array}$ & 0,20 & 0,887 & $\begin{array}{c}\text { Forma de } \\
\text { desplazamiento }\end{array}$ & 0,006 & 0,936 \\
\hline $\begin{array}{l}\text { Tipo de } \\
\text { vivienda }\end{array}$ & 0,719 & 0,869 & $\begin{array}{l}\text { Cuántas comidas } \\
\text { consume al día }\end{array}$ & 4,137 & $0,042^{*}$ \\
\hline $\begin{array}{l}\text { Condición de } \\
\text { vivienda }\end{array}$ & 0,353 & 0,838 & $\begin{array}{l}\text { Beneficiario } \\
\text { restaurante escolar }\end{array}$ & 0,429 & 0,512 \\
\hline $\begin{array}{l}\text { Estado civil de } \\
\text { los padres }\end{array}$ & 0,030 & 0,863 & $\begin{array}{l}\text { Consumo de } \\
\text { cigarrillo }\end{array}$ & 4,464 & $0,035^{*}$ \\
\hline $\begin{array}{l}\text { Nivel educativo } \\
\text { padre }\end{array}$ & 7,116 & $0,008^{*}$ & $\begin{array}{l}\text { Se relaja y disfruta } \\
\text { el tiempo libre }\end{array}$ & 1,290 & 0,256 \\
\hline $\begin{array}{l}\text { Nivel educativo } \\
\text { madre }\end{array}$ & 0,071 & 0,965 & Actividad física & 3,480 & 0,062 \\
\hline
\end{tabular}

Fuente: elaboración propia.

Los resultados del análisis bivariado muestran que la condición física saludable tiene relación de dependencia con el número de comidas al día $(\mathrm{p}=0,042)$, el nivel educativo del padre $(\mathrm{p}=0,035)$ y el consumo de cigarrillos $(\mathrm{p}=0,008)$, observándose que el 76,2\% de los alumnos que mencionaron técnico, tecnológico y universitario como nivel educativo del padre obtuvieron una condición física no saludable, al igual que el $80 \%$ de los escolares que mencionaron consumir cigarrillo o consumir más de tres comidas al día.

Finalmente, al ingresar estas variables al modelo logístico binario realizado, las únicas que mostraron ser predictoras de la variable dependiente condición físi- ca son: el nivel educativo del padre y el consumo de cigarrillo.

Se excluyen del modelo el resto de variables, ya que no mostraron significancia estadística necesaria para configurar el modelo, se aclara que para los análisis del modelo se transformaron las variables participantes en variables dicotómicas o dummys.

Se establece como variable dependiente el estado de condición física general, la cual para efectos del modelo es dicotómica, donde 1 representa a un escolar con condición física saludable y 0 representa un escolar con condición física no saludable, a su vez, se establecen como variables independientes las del nivel 
educativo del padre y del consumo de cigarrillo en los últimos seis meses.

Se observa que la significancia de los betas de la totalidad de las variables modeladas presentan un nivel de significancia estadístico $(\mathrm{p}<0,05)$ (tabla 2 ). Con respecto al valor de los OR se puede afirmar que si existe el hábito del consumo de cigarrillo en un escolar, este tiene 3,356 veces menos posibilidades de tener una condición física saludable, de esta misma manera, para la variable del nivel educativo del padre se puede establecer que a menor nivel educativo de los padres los escolares tienen 2,272 veces más posibilidades de tener una condición física saludable.

Tabla 2. Resultado del modelo de regresión

\begin{tabular}{|c|c|c|c|c|c|c|c|}
\hline & & B & $\begin{array}{c}\text { Error } \\
\text { estándar }\end{array}$ & Wald & gl & Sig. & $\operatorname{Exp}(B)$ \\
\hline \multirow[t]{3}{*}{ Paso 1a } & Cigarrillos & $-1,211$ &, 590 & 4,212 & 1 & ,040 & 3,356 \\
\hline & $\begin{array}{l}\text { Nivel } \\
\text { educativo } \\
\text { padre }\end{array}$ &, 825 & ,309 & 7,126 & 1 & ,008 & 2,282 \\
\hline & Constante & $-2,051$ & ,206 & 99,576 & 1 & ,000 & , 129 \\
\hline
\end{tabular}

Fuente: elaboración propia.

\section{Discusión}

Se evaluaron 339 escolares de Dosquebradas, Risaralda, con un promedio de edad de 14,9+/- 1,96 años, a los cuales se les determinó la condición física a partir de la batería Alpha Fitness, batería utilizada de igual manera en los trabajos realizados por García, et al. (38), Mayorga, et al. (39), Prieto, et al. (29) y Secchi, et al. (40).

Se observó que el transporte activo es el medio de transporte más común en-

126 tre los escolares, siendo el caminar la actividad que más realizan para trans- portarse al colegio $(56,3 \%)$, dato que muestra cierta similitud al ser comparado con los estudios de Rodríguez, et al. (41) quien menciona que el 62,4\% y Gonzales, et al. (42) quien afirma que el $56,8 \%$ de escolares se transportan al colegio de manera activa, sin embargo, Piñeros, et al. (43) en su estudio realizado en cinco ciudades colombianas, reportaron que el 50,3\% del total de escolares evaluados no se trasportan de manera activa al aula de clases (caminar o ir en bicicleta).

El estrato 3 fue el nivel socioeconómico más común en la población participan- 
te $(61,9 \%)$, sin embargo, no se encontró asociación con la condición física ( $\mathrm{p}=$ 0,316 ), al igual que en el trabajo realizado por Gonzales, et al. (27).

Por su parte, en el estudio de Secchi, et al. (40) se observa una mejora de los niveles de condición física con el incremento de la edad, al resaltar cómo los niños con capacidad aeróbica saludable presentan mayores niveles de fuerza en miembros inferiores, un menor índice de masa corporal y de perímetro de cintura, sumado a esto, resalta cómo la prevalencia del sobrepeso y la obesidad fue menor en el grupo con capacidad aeróbica saludable, $22,2 \%$ vs $75,8 \%$ en los participantes masculinos $(\mathrm{p}=0,001)$ y $32,2 \%$ vs $57,9 \%$ en los participantes femeninos $(\mathrm{p}=0,027)$.

Por otro lado, Cano, et al. (6) en un estudio realizado en escolares españoles, identificaron los factores que determinan el nivel de actividad física, encontrándose que el $74 \%$ de la población practica deporte, siendo este el hábito más frecuente a mayor nivel de estudios en los familiares ( $p=0,013)$, en las clases sociales altas $(p=0,021)$ y en el sexo masculino ( $\mathrm{p}=0,001)$; de igual manera, menciona que el porcentaje de niños que pasaba más de tres horas diarias en actividades sedentarias tenía una estrecha relación $(\mathrm{p}=0,01)$ con el nivel educativo de los familiares, al destacar que el 52,9\% de los niños que pasaban más de tres horas en actividades sedentarias, sus familiares tenían o no estudios primarios; sin embargo, lo anterior muestra gran diferencia con los datos encontrados en los escolares dosquebradenses, donde no se encontraron diferencias sig- nificativas entre la práctica de deporte ( $\mathrm{p}$ $=0,676)$, el sexo $(p=0,937)$ y el estrato $(0,316)$ con la condición física saludable, sin embargo, sí se observó asociación significativa con el nivel educativo del padre $(\mathrm{p}=0,008)$, destacándose que el $76,2 \%$ de los alumnos que mencionaron técnico, tecnológico o universitario como nivel educativo del padre, presentaban en mayor proporción una condición física no saludable.

En contraste a lo anterior, Niño, et al. (44) establecen que no existen asociación entre el nivel de estudio de los padres y el nivel de condición física de los hijos, sin embargo, establecen la relación entre el sexo con las diferentes categorías de la condición física, destacando que los hombres presentaron mejores niveles de condición física y las mujeres en la capacidad cardiorrespiratoria, velocidad/agilidad y fuerza $(\mathrm{p}<$ $0,005)$.

A pesar de que el componente cardiorrespiratorio fue el único donde prevaleció el nivel saludable en los escolares $(52,5 \%)$, llama la atención que el rendimiento es menor en esta capacidad al compararse con diferentes regiones, pues la prevalencia de la capacidad aeróbica no saludable en adolescentes argentinos (40) es del 49,1\%; en adolescentes españoles es del 19,3\% para hombres y del $17,3 \%$ para mujeres (45); en adolescentes europeos (46) es del 38\% y $43 \%$ para hombres y mujeres respectivamente; en adolescentes australianos (47) es del $29 \%$ para hombres y $23 \%$ para mujeres; en escolares estadounidenses (48) es del $29 \%$ y $31 \%$ para hombres y mujeres respectivamente. 
Es prioritario pensar que se hace necesario insistir en estrategias que cambien los hábitos de estilo de vida en esta población, ya que el presente estudio muestra que la mayor proporción de escolares presentan una condición física no saludable, al mostrar bajos niveles en tres de los cuatro componentes de la condición física; lo anterior pone tanto a los profesores como a la familia en un rol de promotores de la salud en los jóvenes. De esta manera, tanto el ambiente escolar como el familiar forman un espacio privilegiado, donde se deben promover estilos y hábitos de vida saludable; por tanto, pensamos que es necesario y urgente dar más oportunidades a la actividad física de los adolescentes (acceso fácil a las instalaciones deportivas, bicicarriles para el acceso a centros educativos y de ocio, promoción de la actividad física durante el tiempo de recreo en los centros educativos, promoción de programas extraescolares de ejercicio físico no competitivo, etc.).

Lo anterior debe ser complementado con la generación de procesos y estrategias educativas y de monitoreo frente al comportamiento de la condición física en los escolares de la ciudad, buscando fomentar la formulación de políticas gubernamentales para la distribución de recursos hacia la promoción de los hábitos saludables, de igual manera se recomienda fomentar la práctica de una vida saludable en los padres de familia, dada la influencia que tienen estos últimos frente a la condición física de sus hijos.

Asimismo, es conveniente establecer programas de prevención basados en la educación y eliminación de los factores que predisponen a una condición física no saludable (como el consumo de cigarrillo), disminuyendo así la prevalencia de enfermedades no transmisibles en edades tempranas. En este sentido, estos resultados deben convertirse en piezas importantes en los procesos que permitan mejorar los niveles de condición física en la población escolar de Dosquebradas.

Es indispensable que este tipo de estudios continúen teniendo en cuenta otras variables, tales como el lugar de residencia (rural o urbana), estudios de tipo longitudinal y buscar asociaciones entre los componentes y cada una de las pruebas físicas.

\section{Agradecimientos}

Los autores quieren agradecer a la Universidad Autónoma de Manizales por su colaboración en el desarrollo de la investigación, pero sobre todo a los escolares participantes, ya que sin ellos esto no hubiera sido posible. 


\section{Referencias bibliográficas}

1. Ballesteros, J, Saavedra M, Pérez, $\mathrm{N}$, Villar, Carmen. La estrategia para la nutrición, actividad física y prevención de la obesidad: estrategia NAOS. Rev. Esp. Salud Publica [Internet]. 2007 Oct [citado 2019 Dic 17] ; 81(5): 443-449. Disponible en: http://scielo.isciii.es/scielo.php?script=sci_arttext\&pi$d=S 1135-57272007000500002 \&$ Ing $=e$

2. Malo M. Perspectivas en la lucha contra la obesidad como problema de salud pública. Rev Peru Med Exp Salud Pública [Internet]. 2018 mzo. 23 [citado 2019 febr. 25];34(1):5. Disponible en: https://rpmesp. ins.gob.pe/index.php/rpmesp/article/ view/2760

3. Organización Panamericana de la Salud/Organización Mundial de la Salud. Plan de acción para la prevención de la obesidad en la niñez y la adolescencia [Internet]. 2014 [citado 3 mar 2019]. Disponible en: http://www.paho.org/hq/index. php?option=com_docman\&task $=$ doc view\&ltemid=270\&gid=28899\&lang=es

4. Waters E, da Silva A, Burford B, Brown T, Campbell K, Gao Y, et al. Interventions for preventing obesity in children. Cochrane Database Syst Rev. 2011 dic. 7;12:CD001871.

5. OMS. Subsanar las desigualdades en una generación. Informe de la Comisión de Determinantes Sociales en Salud. OMS; 2009.

6. Cano A, Pérez I, Casares I, Alberola S. Determinantes del nivel de actividad física en escolares y adolescentes: estudio OPACA. An Pediatría. 2011;74(1):15-24.

7. Kraus W, Houmard J, Duscha B, Knetzger K, Wharton M, McCartney J, et al. Effects of the amount and intensity of exercise on plasma lipoproteins. N Engl J Med. 2002 nov. 7;347(19):1483-92.

8. Myers J, Prakash M, Froelicher V, Do D, Partington S, Atwood JE. Exercise capacity and mortality among men referred for exercise testing. N Engl J Med. 2002 mzo. 14;346(11):793-801.

9. Leiva AM, Petermann F, Martínez MA, Troncoso C, Concha Y, Garrido A, et al. Asociación de un índice de estilos de vida saludable con factores de riesgo cardiovascular en población chilena. Rev Med Chil [Internet]. 2018 dic. [citado 2019 febr. 26];146(12):1405-14. Disponible en: http:// www.scielo.cl/scielo.php?script=sci_arttext\&pid=S0034-98872018001201405\&I$\mathrm{ng}=\mathrm{en} \& \mathrm{nrm}=$ iso\&tlng=en

10. Healy GN, Dunstan DW, Salmon J, Shaw JE, Zimmet PZ, Owen N. Television time and continuous metabolic risk in physically active adults. Med Sci Sports Exerc [Internet]. 2008 abr. [citado 2019 febr. 26];40(4):639-45. Disponible en: http:// www.ncbi.nlm.nih.gov/pubmed/18317383

11. Kvaavik E, Tell G, Klepp K-I. Predictors and tracking of body mass index from adolescence into adulthood. Arch Pediatr Adolesc Med. 2003 dic. 1;157(12):1212.

12. Ramírez J, Mayorga D, Mompeán M. Cumplimiento de los estándares curriculares de condición física-salud en educación física: Estudio de la planificación en la formación inicial. Cult Cienc y Deport [Internet]. 2016 [citado 2019 febr. 26];11(32):8795. Disponible en: https://www.redalyc. org/pdf/1630/163046240002.pdf

13. Dollman J, Norton K, Norton L. Evidence for secular trends in children's physical activity behaviour. $\mathrm{Br} \mathrm{J}$ Sports Med. 2005 dic. 1;39(12):892-7.

14. Argüelles F. Tratado de gastroenterología, hepatología y nutrición pediátrica aplicada de la SEGHNP [Internet]. 2011 [citado 2019 febr. 27]. 976 p. Disponible en: www.cedro.org

15. Mauro I, Megías A, De Angulo B, Bodega P, Rodríguez P, Grande G, et al. Influencia de hábitos saludables en el estado ponderal de niños y adolescentes en edad escolar. Nutr Hosp [Internet]. 2015 
[cited 2019 febr. 27];31(5):1996-2005. Disponible en: http://scielo.isciii.es/pdf/ nh/v31n5/12originalobesidad04.pdf

16. Gómez R. La enseñanza de la educación física en el nivel inicial y el primer ciclo de EGB. Buenos Aires: Editorial Stadium; 2002.

17. Skinner J, Bounds W, Carruth B, Morris M, Ziegler P. Predictors of children's body mass index: a longitudinal study of diet and growth in children aged 2-8 y. Int J Obes. 2004 abr. 2;28(4):476-82.

18. González M, Mejía E, Pacheco A, González L, Cuellar C, Castillo L, et al. Cualidades físicas del adulto mayor activo de la ciudad de Tunja. Rev Investig en Salud Univ Boyacá [Internet]. 2017 [citado 2019 febr. 27];3(1):33. Disponible en: http://revistasdigitales. uniboyaca.edu.co/index.php/rs/article/ view/140/135

19. Castillo MJ. La condición física es un componente importante de la salud para los adultos de hoy y del mañana. Selección [Internet]. 2007 [citado 2019 febr. 27];17(1):2-8. Disponible en: https://www.cafyd.com/selec0701/Selultimo.pdf

20. Sacchetti R, Ceciliani A, Garulli A, Masotti A, Poletti G, Beltrami P, et al. Physical fitness of primary school children in relation to overweight prevalence and physical activity habits. J Sports Sci. 2012 abr;30(7):633-40.

21. Monteiro M, Amaral T, Oliveira B, Borges N. Protective effect of physical activity on dissatisfaction with body image in children - A cross-sectional study. Psychol Sport Exerc. 2011;12(5):563-9.

22. Sobal J, Stunkard AJ. Socioeconomic status and obesity: A review of the literature. Psychol Bull. 1989 mzo;105(2):260-75.

23. Padrón H. Los determinantes sociales, las desigualdades en salud. Rev Cuba Salud Pública. 2010;37:136-44.
24. Palomino C, González J, Ramos C. Body composition and physical fitness in Colombian secondary school and half of Ibagué. Biomedica. 2017 sept. $1 ; 37(3): 408$.

25. Cuenca $M$, Jiménez $D$, España $V$, Artero E, Castro J, Ortega F, et al. Condición física relacionada con la salud y hábitos de alimentación en niños y adolescentes: propuesta de addendum al informe de salud escolar. Rev Investig en Educ. 2011;9(2):35-50.

26. Guillén F, Ramírez M. Relación entre el autoconcepto y la condición física en alumnos del tercer ciclo de primaria. Rev Psicol del Deport [Internet]. 2011 [citado 2018 sept. 30];20(1):45-59. Disponible en: http://www.redalyc.org/articulo. oa?id=235119302005

27. González E, Ortega A. Relación de sobrepeso y obesidad con nivel de actividad física, condición física, perfil psicomotor y rendimiento escolar en población infantil (8 a 12 años) de Popayán. Rev Mov Científico. 2013;7(1):71-84.

28. Arriscado D, Muros J, Zabala M, Dalmau J. Relación entre condición física y composición corporal en escolares de primaria del norte de España (Logroño). Nutr Hosp. 2014;30(2):385-94.

29. Prieto D, Correa J, Ramírez R. Niveles de actividad física, condición física y tiempo en pantallas en escolares de Bogotá, Colombia: Estudio Fuprecol. Nutr Hosp. 2015;32(5):2184-92.

30. Rosa A, Rodríguez P, García E, Pérez J. Niveles de condición física de escolares de 8 a 11 años en relación al género ya su estatus corporal. Ágora para la Educ física y el Deport. 2015;17(3):237-50.

31. Salleg M, Petro J. Perfil de aptitud física de los escolares de 12 a 18 años del municipio de Montería. 2010; 15: 145. Disponible en https://www.efdeportes. com/efd149/aptitud-fisica-de-los-escolares.htm 
32. Artero E, España V, Castro J, Ruiz J, Jiménez D, Aparicio V, et al. Criterion-related validity of field-based muscular fitness tests in youth. J Sports Med Phys Fitness. 2012 oct. 1;52(3):263-72.

33. Ruiz J, España V, Castro J, Artero E, Ortega F, García M, et al. Batería ALPHA-Fitness : test de campo para la evaluación de la condición física relacionada con la salud en niños y adolescentes. Nutr Hosp. 2011;26(6):1210-5.

34. Moro P, Castillo M, De Espinosa M, Algaba E, López N, Serrano M. Semilongitudinal analysis of the physical status in madrilenian adolescents. Arch Med del Deport. 2016;33(3):183-92.

35. Ministerio de Salud. Resolución 8430 de 1993. Ministerio de Salud y Protección Social, República de Colombia 1993, p. 1-19.

36. Manzini, J. Declaración de Helsinki: principios éticos para la investigación médica sobre sujetos humanos. Acta bioeth. [Internet]. 2000 Dic [citado 2019 mar 16] ; 6( 2 ): 321-334. Disponible en https:// scielo.conicyt.cl/scielo.php?script=sci_arttext\&pid=S1726-569X2000000200010\%\&ng=es.

\section{S1726-569X2000000200010}

37. OMS. Recomendaciones mundiales sobre actividad física para la salud. 2010. p. 58.

38. García A, Burgueño R, López D, Ortega F. Condición física, adiposidad y autoconcepto en adolescentes. Estudio piloto. Rev Psicol del Deport. 2013;22(2):453-61.

39. Mayorga D, Merino R, Rodríguez E. Relación entre la capacidad cardiorrespiratoria y el rendimiento en los tests de condición física relacionada con la salud incluidos en la batería ALPHA en niños de 10-12 años. CCD Cult. 2013;8(22):41-7.

40. Secchi J, García G, España V, Castro J. Condición física y riesgo cardiovascular futuro en niños y adolescentes argentinos: una introducción de la batería ALPHA. Arch Argent Pediatr. 2014;112(2):132-40. 41. Rodríguez C, Villa E, Pérez I, Delgado M, Ruiz J, Chillón P. Los factores familiares influyen en el desplazamiento activo al colegio de los niños españoles. Nutr Hosp [Internet]. 2013 [citado 2018 en. 28];28(3):756-63. Disponible en: http://www.nutricionhospitalaria.com/ pdf/6399.pdf

42. González V, Rodríguez C, Huertas D, Torecedor P, Ruiz J, Chillon P. Factores personales y ambientales asociados con el desplazamiento activo al colegio de los escolares españoles. Rev Psicol del Deport. 2011;21(2):343-9.

43. Piñeros M, Pardo C. Actividad física en adolescentes de cinco ciudades colombianas: resultados de la Encuesta Mundial de Salud a Escolares. Rev Salud Pública. 2010;12(6):903-14.

44. Niño C, Guijarro M, Martínez M, Gómez R, Ruiz S, Palencia N. Relación entre nivel de estudios de los padres y condición física de los escolares. Diferencias de género. Rev Andaluza Med del Deport. 2015 mzo. 1;8(1):46.

45. Ortega F, Ruiz J, Castillo M, Moreno L, González M, Wärnberg J, et al. Low level of physical fitness in Spanish adolescents. Relevance for future cardiovascular health (AVENA study). Rev Esp Cardiol. 2005 ag.;58(8):898-909.

46. Ortega F, Artero E, Ruiz JR, Espana V, Jimenez D, Vicente G, et al. Physical fitness levels among European adolescents: the HELENA study. $\mathrm{Br} \mathrm{J}$ Sports Med. 2011 en. 1;45(1):20-9.

47. Catley M, Tomkinson G. Normative health-related fitness values for children: analysis of 85347 test results on 9-17 year old Australians since 1985. Br J Sports Med. 2013 en.;47(2):98-108.

48. Secchi J, García G. Aptitud física cardiorrespiratoria y riesgo cardiometabólico en personas adultas jóvenes. Rev Esp Salud Pública. 2013;87:35-48. 